\title{
A Coordinated Search Algorithm for a Lost Target on the Plane
}

\author{
Sundus Naji Al-Aziz ${ }^{1, *}$, Abd Al-Aziz Hosni El-Bagoury ${ }^{2}$ and W. Afifi, ${ }^{2,3}$ \\ ${ }^{1}$ Department of Mathematics, College of Science, Princess Nourah bint Abdulrahman University, Riyadh, Saudi Arabia \\ ${ }^{2}$ Department of Mathematics, Faculty of Science, Tanta University, Tanta, Egypt \\ ${ }^{3}$ Department of Mathematics and Statistics, College of Science, Taibah University, Yanbu, Saudi Arabia \\ ${ }^{*}$ Corresponding Author: Sundus Naji Al-Aziz. Email: Sundus.alaziz3@gmail.com \\ Received: 18 December 2020; Accepted: 20 April 2021
}

\begin{abstract}
Concepts in search theory have developed since World War II. The study of search plans has found considerable interest among searchers due to its wide applications in our life. Searching for lost targets either located or moved is often a time-critical issue, especially when the target is very important . In many commercial and scientific missions at sea, it is of crucial importance to find lost targets underwater. We illustrate a technique known as coordinated search, that completely characterizes the search for a randomly located target on a plane. The idea is to avoid wasting time looking for a missing target. Two searchers or robots start from the center of a circle to search out a lost target, the first searcher looks for the target on the right side of the circular area, and the second one looks for it on the left side. The time taken to detect the target is obtained by assuming the target's position has a symmetric distribution. The procedures to facilitate the detection of the target are presented as an algorithm and as a flowchart. An application demonstrates the applicability of this search technique and the associated decrease in search cost. Its effectiveness is illustrated by numerical results, which indicates considerable promise.
\end{abstract}

Keywords: Expected value; sensors; lost target; coordinated search; search algorithm; lost ship

\section{Introduction}

Search theory rests on the oldest area of operations research. The initial steps made by Koopman in the Anti-submarine Warfare Operations Research Group of the U.S. Navy during World War II for submarine detection are still widely used for this purpose. The theory has also been used by the Navy to search for objects such as the H-bomb lost in the ocean near Palomares, Spain, in 1966, the submarine Scorpion lost in 1968, and numerous lesser-known objects. The U.S. Coast Guard uses the search theory to plan some of its more complicated search and rescue efforts.

To mention some important models of search plans, we should begin with the linear search strategy, which has many life and mission applications, such as searching for a damaged unit in a large linear system (electrical power lines, telephone lines, and gas support lines), whether a linear system is independent or intersecting (see El-Rayes et al. [1], Mohamed [2], Mohamed et al. [3-5], Balkhi [6]).

This work is licensed under a Creative Commons Attribution 4.0 International License, which permits unrestricted use, distribution, and reproduction in any medium, provided the original work is properly cited. 
The coordinated search technique has been studied in the case of a linear search. Mohamed et al. [7-9] discussed the coordinated search technique for a target on two intersecting lines, where the target has a symmetric and asymmetric distribution (See Mohamed et al. [10], Afifi et al. [11]). More advanced work applied the coordinated search technique to a moving target on one line as well as on many independent lines. The coordinated search technique in the plane was discussed by Mohamed et al. [12]. El-Hadidy et al. $[13,14]$ proposed a model in $3-\mathrm{D}$ space to find a randomly located target by one or two searchers. Caraballo et al. [15] considered a model in search theory to find a randomly located target in 3-D space. Kassem et al. [16] studied a more sophisticated search model in 3-D space to find a randomly located target. More recently, Teamah et al. [17] obtained an optimal discrete search for randomly moving COVID-19 between several cells in the human body using a monitoring system. Afifi et al. [18] studied the existence of the multi-generalized linear search problem to detect a randomly located target in one of several real lines.

We present a coordinated algorithm, with the important advantage that it avoids wasting time searching for the target. The mission is carried out by two searchers starting from the origin of a known circular area on the sea surface. The first searcher looks for the target on the right side of the circular area, and the second one looks for it on the left side. It is important to note that neither searcher returns to the origin. We use modern means of communication and sign language to save much effort and time.

\section{Problem Formulations}

On May 19, 2020, maritime authorities announced that they had lost contact with a small ship on its way to the Socotra Archipelago port in the Indian Ocean, carrying two families in addition to the ship's crew. They suddenly received a call from the missing ship, which enabled them to determine its coordinates. Navy ships were then sent out to search for the missing ship. We present a coordinated algorithm to solve this kind of problem, with the advantage of a detection time saving element, as the probability distribution function of the ship's location is known to the searchers (sensors).

\subsection{Search Framework}

Search space: Circular two-dimensional area.

Target: The target is a lost ship randomly located on the sea surface.

Means of search: The search is performed by two searchers on a circular section. They start looking together from the point $(0,0)$ (the center of the circular area), where the region is divided into right and left parts. We also divide the area into concentric circles, as in Fig. 1.

\subsection{The Searching Technique}

In this model, the search for the missing ship is carried out according to coordinated movement between the two searchers. After each move, both send signals to a marine ship's signal reception center by radio telex. There are two types of detection:

1. Perfect detection: One of the searchers detects the lost ship in the specified search section and sends a positive sign.

2. False detection: Neither searcher detects the lost ship in the specified search section, and one or both sends a positive sign.

Let $(\mathrm{X}, \mathrm{Y})$ be independent random variables representing the position of the target, with cumulative distribution function (CDF) $F(x, y)$ and probability density function (PDF) $f(x, y)$.

Searchers $s_{1}$ and $s_{2}$ start searching together for the lost ship (target) from the origin $(0,0)$ with equal speeds $v_{1}=v_{2}=1$, and they search with a regular speed on the sectors and their tracks. Searcher $\mathrm{s}_{1}$ 
searches on the right side, and searcher $\mathrm{s}_{2}$ on the left side. Searcher $\mathrm{s}_{1}$ goes through a + ve part $y$-axis with a distance $\mathrm{r}_{1}$ (radius of the first circle) and starts searching sector $\mathrm{L}_{1}$. After searching sector $\mathrm{L}_{1}$, the searcher reaches the y-axis again but does not return to the origin point and moves toward sector $L_{2}$ with distance $\mathrm{r}_{2}-\mathrm{r}_{1}$. When it completes the search of sector $L_{2}$ and does not find the target, it continues searching sector L3, and so on. Simultaneously, searcher $\mathrm{s}_{2}$ searches the opposite area on the left hand-side, goes through a -ve part y-axis with distance $\mathrm{r}_{1}$, and starts searching sector $\mathrm{M}_{1}$. After searching this sector and not finding the target, it moves to sector $M_{2}$ with distance $r_{2}-r_{1}$. When it completes searching sector $M_{2}$ and does not find the target, it continues searching sector $M_{3}$, and so on. Searchers $\mathrm{s}_{1}$ and $\mathrm{s}_{2}$ search sectors $\mathrm{L}_{1}$ and $\mathrm{M}_{1}$ at the same time. If one of them detects the lost target, it will send a positive sign and the search will end. If both send negative signs, then the two searchers complete their search and move to the next sectors, $L_{2}$ and $M_{2}$, and so on, until one of the searchers detects the lost ship. The searchers do not return to the origin so as to save search time and reduce the expected time to detect the target.

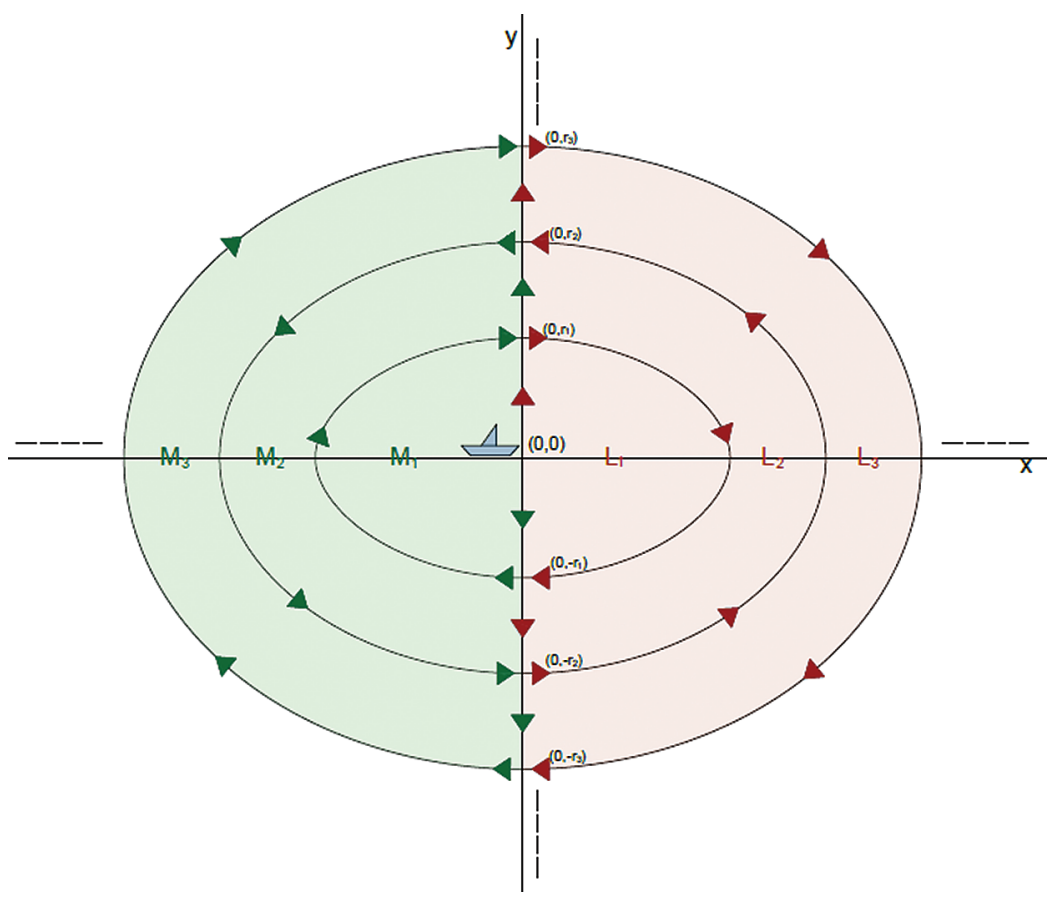

Figure 1: The search path

Any track has width $r_{i}-r_{i-1}$. The searcher moves on the y-axis $+v e$ and $-v e$ sides until it detects the target. We aim to calculate the expected length of time to detect the target and search the optimal search plan.

Searchers $\mathrm{s}_{1}$ and $\mathrm{s}_{2}$ follow a coordinated search path to find the target. Let $\mathrm{E}_{\mathrm{i}}$ and $\mathrm{F}_{\mathrm{i}}$ be the search paths of $s_{1}$ and $s_{2}$, respectively, $i=1,2, \ldots, n$, and let $L_{i}$ and $M_{i}$ be their respective search sectors. Let $t_{1}$ and $t_{2}$ be the respective times taken by $s_{1}$ and $t_{2}$ to search each sector in paths $E_{i}$ and $F_{i}$, respectively.

The search proceeds as follows.

Step 1: The two searchers move from $(0,0)$ to detect the target. Searcher $s_{1}$ follows search path $e_{1}$ as follows: Searcher $\mathrm{s}_{1}$ goes to $\left(0, r_{1}\right)$ with distance $\left|r_{1}\right|$ through a +ve part in y-axis. If the target is not found, it completely searches sector $\mathrm{L}_{1}$ and continues tracking until the point $\left(0,-r_{1}\right)$. At the same time, searcher $\mathrm{s}_{2}$ goes to $\left(0,-r_{1}\right)$ with distance $\left|r_{1}\right|$ through a -ve part in $\mathrm{x}$-axis. If the target is not found, it completely searches sector $\mathrm{M}_{1}$ and continues tracking until the point $\left(0, r_{1}\right)$. At this time, the two searchers send signals 
to a marine ship's signal reception center. If one of them sends a positive signal, then the search ends; however, if the two searchers send a negative signal, the searchers will move on to the following step.

Step 2: Searcher $\mathrm{s}_{1}$ completes the search for the lost target and moves to the point $\left(0, \mathrm{r}_{2}\right)$ with a distance $\left|\mathrm{r}_{2}-\mathrm{r}_{1}\right|$. The first searcher follows the search path $e_{2}$ as follows: searcher $\mathrm{s}_{1}$ goes to $\left(0, r_{2}\right)$ with distance $\left|\mathrm{r}_{2}-\mathrm{r}_{1}\right|$ through a + ve part on the y-axis. If the target is not found, it completely searches sector $L_{2}$ and keeps tracking until the point $\left(0,-r_{2}\right)$. At the same time, searcher $\mathrm{s}_{2}$ goes to $\left(0,-r_{2}\right)$ with distance $\left|\mathrm{r}_{2}-\mathrm{r}_{1}\right|$ through a -ve part on the x-axis. If the target is not found, it completely searches sector $M_{2}$ and keeps tracking until the point $\left(0, r_{2}\right)$. At this time, the two searchers send signals to a marine ship's signal reception center. If one of them sends a positive signal, then the search ends; however, if the two searchers send a negative signal, the searchers will move on to the following step.

Step 3: Searcher $\mathrm{s} 1$ completes the search for the lost target and moves to the point $\left(0, \mathrm{r}_{3}\right)$ with a distance $\left|r_{3}-r_{2}\right|$. The first searcher follows the search path $e_{3}$ as follows: searcher $s_{1}$ goes to $\left(0, r_{3}\right)$ with distance $\left|\mathrm{r}_{3}-\mathrm{r}_{2}\right|$ through a + ve part on the y-axis. If the target is not found, it completely searches sector $L_{2}$ and keeps tracking until the point $\left(0,-r_{2}\right)$. At the same time, searcher $\mathrm{s}_{2}$ goes to $\left(0,-r_{3}\right)$ with distance $\left|\mathrm{r}_{3}-\mathrm{r}_{2}\right|$ through the -ve part on the x-axis. If the target is not found, it completely searches sector $M_{3}$ and keeps tracking until the point $\left(0, r_{3}\right)$. At this time, the two searchers send signals to a marine ship's signal reception center. If one of them sends a positive signal, then the search ends; however, if the two searchers send a negative signal, the searchers will move on to the next sectors, and so on, until one of the two searchers detects the lost ship.

The searchers $s_{1}$ and $s_{2}$ follow the search path. Let $E_{i}$ be the path for the first searcher, where $i \geq 0, t_{1}$ is the time for the first searcher, $L_{i}$ is the sector which $s_{1}$ searches, $i=1,2, \ldots, n$, and $F_{i}$ the path of the second searcher, where $i \geq 0, M_{i}$ is the sector which $s_{2}$ searches, $i=1,2, \ldots . n$, and the two searchers move from $(0,0)$ to detect the target. The first search path $\mathrm{e}_{1}$ of $\mathrm{s}_{1}$ is as follows: searcher $\mathrm{s}_{1}$ goes to $\left(0, r_{1}\right)$ with distance $\left|r_{1}\right|$ through a + ve part on the y-axis. If the target is not found, it completely searches sector $\mathrm{L}_{1}$ and keeps tracking until the point $\left(0,-r_{1}\right)$. The time taken in this step is $\mathrm{t}_{1}=\left|\mathrm{r}_{1}\right|+\frac{\pi}{\mathrm{w}_{1}}$, and the searcher moves to the point $\left(0, r_{2}\right)$ with distance $\left|r_{2}-r_{1}\right|$. It takes time $t_{1}=\left|r_{2}-r_{1}\right|+\frac{\pi}{w_{2}}$ if it does not find the target and moves on to sector $L_{2}$ while continuing to track. If the target is not found, it moves toward $\left(0, r_{3}\right)$ to search sector $\mathrm{L}_{3}$ and keeps tracking until the point $\left(0,-\mathrm{r}_{3}\right)$ with distance $\left|\mathrm{r}_{3}-\mathrm{r}_{2}\right|$; it takes time $\mathrm{t}_{1}=\left|\mathrm{r}_{3}-\mathrm{r}_{2}\right|+\frac{\pi}{\mathrm{w}_{3}}$, and so on. The number of circles is $\mathrm{i}=1,2, \ldots$ These circles are divided into two equal two sectors, $\mathrm{L}_{\mathrm{i}}$ and $\mathrm{M}_{\mathrm{i}}$.

Searcher $\mathrm{s}_{2}$ goes to $\left(0,-r_{1}\right)$ with distance $\left|r_{1}\right|$ through a -ve part on the $\mathrm{x}$-axis. If the target is not found, it completely searches sector $\mathrm{M}_{1}$ and continues tracking until the point $\left(0, r_{1}\right)$ with time $\mathrm{t}_{2}=\left|\mathrm{r}_{1}\right|+\frac{\pi}{\mathrm{w}_{1}}$, then moves to the point $\left(0, r_{2}\right)$ with distance $\left|r_{2}-r_{1}\right|$ and takes time $t_{2}=\left|r_{2}-r_{1}\right|+\frac{\pi}{w_{2}}$. If the target is not found, it searches sector $\mathrm{M}_{2}$ until the point $\left(0, r_{2}\right)$. If the target is not found, it moves toward $\left(0,-\mathrm{r}_{3}\right)$, searches sector $\mathrm{M}_{3}$, and keeps tracking until the point $\left(0, \mathrm{r}_{3}\right)$ with distance $\left|\mathrm{r}_{3}-\mathrm{r}_{2}\right|$, and so on. 


\section{Expected Time to Detect Target}

Theorem 1: The expected value of the time to detect the target is

$$
\begin{aligned}
& E\left(t_{\phi}\right)=2\left[\left[\left(\left|r_{1}\right|+\frac{\pi}{w_{1}}\right) \int_{0}^{\frac{\pi}{2}} \int_{0}^{r_{1}} g_{1}(r, \theta) r d r d \theta\right]+\left[\left(\left|r_{2}-r_{1}\right|+\frac{\pi}{w_{2}}\right) \int_{0}^{\frac{\pi}{2}} \int_{r_{1}}^{r_{2}} g_{2}(r, \theta) r d r d \theta\right]\right. \\
& +\left[\left(\left|r_{3}-r_{2}\right|+\frac{\pi}{w_{3}}\right) \int_{0}^{\frac{\pi}{2}} \int_{r_{2}}^{r_{3}} g_{3}(r, \theta) r d r d \theta\right]+\cdots \cdots+\left[\left(\left|r_{n}-r_{n-1}\right|+\frac{\pi}{w_{n}}\right) \int_{0}^{\frac{\pi}{2}} \int_{r_{n-1}}^{r_{n}} g_{n}(r, \theta) r d r d \theta\right] \\
& =2\left[\left[\left(\left|r_{i}-r_{i-1}\right|+\frac{\pi}{w_{i}}\right) \int_{0}^{\frac{\pi}{2}} \int_{r_{i-1}}^{r_{i}} g_{i}(r, \theta) r d r d \theta\right]\right]
\end{aligned}
$$

where $\mathrm{i}=1,2,3, \ldots, \mathrm{n}, r_{0}=0$.

Proof:

1) For $s_{1}$ :

If the target is in sector L1, then $t_{1}=\left|r_{1}\right|+\frac{\pi}{w_{1}}$.

If the target is in sector L2, then $t_{1}=\left|r_{2}-r_{1}\right|+\frac{\pi}{w_{2}}$.

If the target is in sector L3, then $t_{1}=\left|r_{3}-r_{2}\right|+\frac{\pi}{w_{3}}$, and so on.

2) For $s_{2}$ :

If the target is in sector M1, then $t_{2}=\left|r_{1}\right|+\frac{\pi}{w_{1}}$.

If the target is in sector $\mathrm{M} 2$, then $t_{2}=\left|r_{2}-r_{1}\right|+\frac{\pi}{w_{2}}$.

If the target is in sector M3, then $t_{2}=\left|r_{3}-r_{2}\right|+\frac{\pi}{w_{3}}$, and so on.

Then

$$
\begin{aligned}
& E\left(t_{\phi}\right)=\left[\left(\left|r_{1}\right|+\frac{\pi}{w_{1}}\right) \int_{0}^{\frac{\pi}{2}} \int_{0}^{r_{1}} g_{1}(r, \theta) r d r d \theta\right]+\left[\left(\left|r_{2}-r_{1}\right|+\frac{\pi}{w_{2}}\right) \int_{0}^{\frac{\pi}{2}} \int_{r_{1}}^{r_{2}} g_{2}(r, \theta) r d r d \theta\right]+\cdots \\
& {\left[\left(\left|r_{3}-r_{2}\right|+\frac{\pi}{w_{3}}\right) \int_{0}^{\frac{\pi}{2}} \int_{r_{2}}^{r_{3}} g_{3}(r, \theta) r d r d \theta\right]+\cdots \cdots+\left[\left(\left|r_{n}-r_{n-1}\right|+\frac{\pi}{w_{n}}\right) \int_{0}^{\frac{\pi}{2}} \int_{r_{n-1}}^{r_{n}} g_{n}(r, \theta) r d r d \theta\right]+} \\
& {\left[\left(\left|r_{1}\right|+\frac{\pi}{w_{1}}\right) \int_{0}^{\frac{\pi}{2}} \int_{0}^{r_{1}} g_{1}(r, \theta) r d r d \theta\right]+\left[\left(\left|r_{2}-r_{1}\right|+\frac{\pi}{w_{2}}\right) \int_{0}^{\frac{\pi}{2}} \int_{r_{1}}^{r_{2}} g_{2}(r, \theta) r d r d \theta\right]+} \\
& {\left[\left(\left|r_{3}-r_{2}\right|+\frac{\pi}{w_{3}}\right) \int_{0}^{\frac{\pi}{2}} \int_{r_{2}}^{r_{3}} g_{3}(r, \theta) r d r d \theta\right]+\cdots \cdots+\left[\left(\left|r_{n}-r_{n-1}\right|+\frac{\pi}{w_{n}}\right) \int_{0}^{\frac{\pi}{2}} \int_{r_{n-1}}^{r_{n}} g_{n}(r, \theta) r d r d \theta\right]} \\
& =2\left[\left[\left(\left|r_{i}-r_{i-1}\right|+\frac{\pi}{w_{i}}\right)_{0}^{\frac{\pi}{2}} \int_{0}^{r_{r_{i-1}}} g_{i}(r, \theta) r d r d \theta\right]\right]
\end{aligned}
$$

\section{Algorithm and Flowchart}

The calculation of the expected value of the time to detect the lost target is described in the following algorithm and flowchart. 
- Main algorithm

- initialize searchers $\mathbf{S}_{\mathbf{1}}$ and $\mathbf{S}_{\mathbf{2}}$ to start point $(0,0)$;

- Velocities $\mathbf{V}_{\mathbf{1}}=\mathbf{V}_{\mathbf{2}}=1$;

- $\mathrm{i}=1$;

- $\mathbf{r}_{\mathbf{i}-1}=\mathbf{0}$;

- for (i to $\mathrm{k}$ )

- distance $\mathbf{D}=\left|\mathbf{r}_{\mathbf{i}}-\mathbf{r}_{\mathbf{i}-1}\right|$;

- if $(\mathrm{i}=$ odd number $)$ then

- move S1 toward point $\left(0, r_{i}\right)$ with distance $\mathbf{D} \& \&$ move $S 2$ toward point $\left(0,-r_{\mathbf{i}}\right)$ with distance $\mathbf{D}$;

- $\mathbf{S} 1$ completes searching in sector $\mathbf{L i}$ with same distance $\mathbf{D}$ and tracks until point $\left(0,-\mathbf{r}_{\mathbf{i}}\right) \& \&$

S2 completes searching in sector Mi with same distance $\mathbf{D}$ and tracks until point $\left(0, \mathrm{r}_{\mathbf{i}}\right)$;

- else

- move S1 toward point $\left(0,-r_{\mathbf{i}}\right)$ with distance $\mathbf{D} \& \&$ move $S 2$ toward point $\left(0, r_{\mathbf{i}}\right)$ with distance $\mathbf{D}$;

- $\mathrm{S} 1$ completes searching in sector $\mathbf{L i}$ with same distance $\mathbf{D}$ and tracks until point $\left(0, \mathrm{r}_{\mathbf{i}}\right) \& \&$

S2 completes searching in sector $\mathbf{M i}$ with same distance $\mathbf{D}$ and tracks until point $\left(0,-\mathbf{r}_{\mathbf{i}}\right)$;

- end if

- calculate time for step $\mathbf{T}_{\mathbf{i}}=$ distance $\mathbf{D}+\left(3.14 / \mathrm{w}_{\mathbf{i}}\right)$

- if (target not found in S1 || S2 ) then

- print $\mathrm{S} 1+$ "not found target in" $+\mathrm{L}_{\mathbf{i}}$ )

- print $\mathrm{S} 2+$ "not found target in" + $\mathrm{M}_{\mathbf{i}}$ )

- else

- if (target found in S1) then

- print green signal+S1+ "found target in" $+\mathbf{L}_{\mathbf{i}}+$ "time:" $+\mathbf{T}_{\mathbf{i}}$

- else

- print green signal+S2+ "found target in" $+\mathbf{M}_{\mathbf{i}}+$ "time:" $+\mathbf{T}_{\mathbf{i}}$

- exit a for loop

- end if

- End for

- End Main 
CSSE, 2022, vol.40, no.1

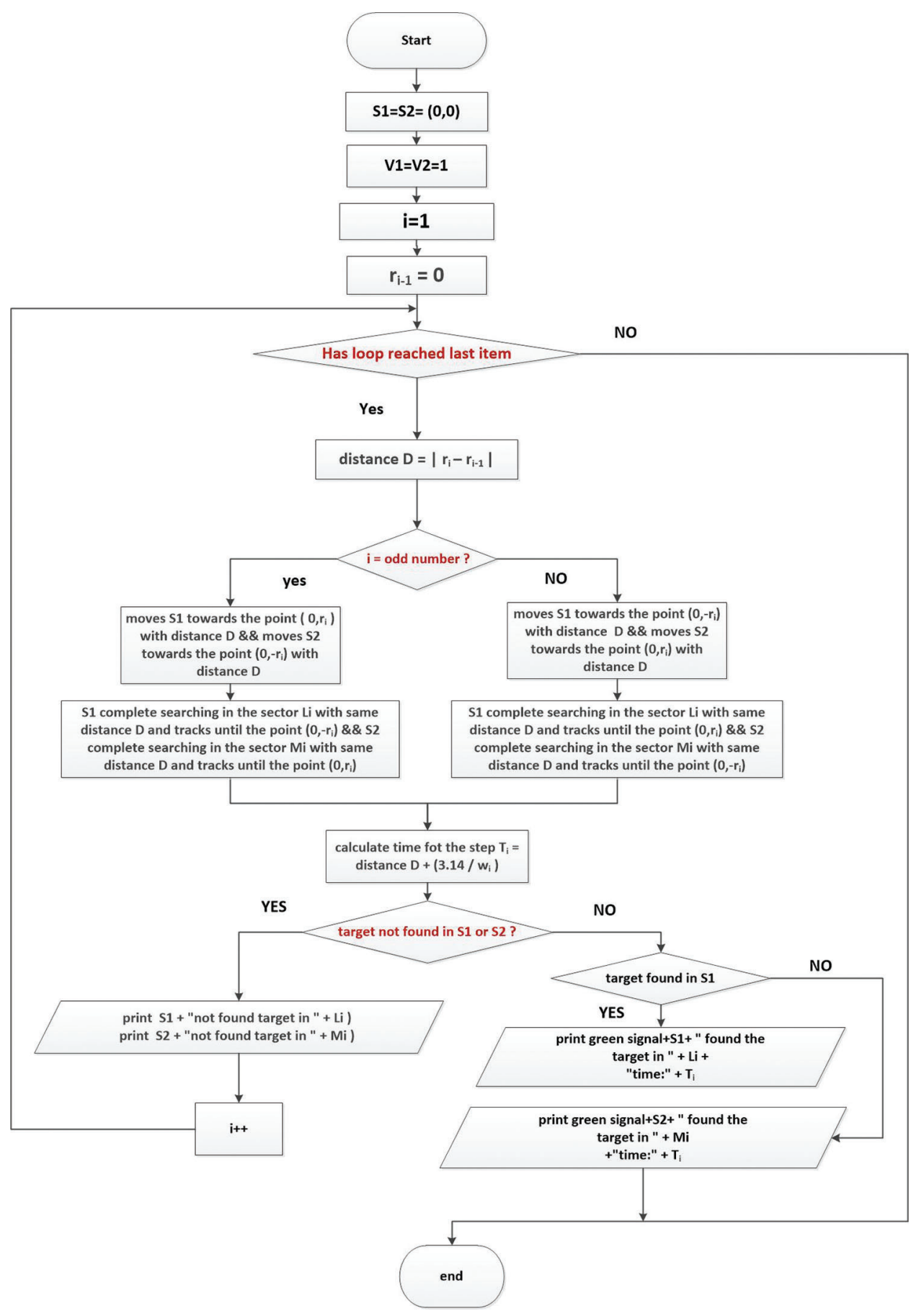




\section{Application}

Searchers $s_{1}$ and $s_{2}$ have a mission to search for a lost ship on the sea surface. The ship's location follows a standard bivariate normal distribution for two independent random variables $x, y, f(x, y)=\frac{1}{2 \pi} e^{\frac{-x^{2}+y^{2}}{2}}$. Changing to polar coordinates $x=r \cos \theta, y=r \sin \theta$, the Jacobian matrix is defined as

$J(u, v)=\operatorname{det}\left(\begin{array}{ll}\frac{\partial x}{\partial r} & \frac{\partial x}{\partial \theta} \\ \frac{\partial y}{\partial r} & \frac{\partial y}{\partial \theta}\end{array}\right)=J(u, v)=\operatorname{det}\left(\begin{array}{cc}\cos \theta & -r \sin \theta \\ \sin \theta & r \cos \theta\end{array}\right)=r \cos ^{2} \theta+r \sin ^{2} \theta=r\left(\cos ^{2} \theta+\sin ^{2} \theta\right)=r$. will be

So, $g(r, \theta)=\frac{1}{2 \pi} e^{\frac{-r^{2}}{2}}$, and by substituting in Eq. (1), the expected value of the time to detect the target $E\left(t_{\phi}\right)=2\left[\left(\left|r_{i-} r_{i-1}\right|+\frac{\pi}{w_{i}}\right) \int_{0}^{\frac{\pi}{2}} \int_{r_{i-1}}^{r_{i}} \frac{1}{2 \pi} r e^{\frac{-r^{2}}{2}} r d r d \theta=\int_{0}^{\frac{\pi}{2}} \int_{r_{i-1}}^{r_{i}} r^{2} e^{-\frac{r^{2}}{2}} d r d \theta=\frac{\pi}{2} \int_{r_{i-1}}^{r_{i}} r^{2} e^{-\frac{r^{2}}{2}} d r\right.$

The first search if $\mathrm{r}: 0 \rightarrow r_{1}$; then $E\left(t_{\phi}\right)=\frac{\pi}{2}\left[\frac{e^{-\frac{r_{1}^{2}}{2}\left(\sqrt{\pi} e^{\frac{r_{1}{ }^{2}}{2}} \operatorname{erf} \frac{r_{1}}{\sqrt{2}}-\sqrt{2} r_{1}\right)}}{\sqrt{2}}\right]$

The Second search if $\mathrm{r}: r_{1} \rightarrow r_{2}$; then the expected value will be

$=\frac{\pi}{2}\left[\frac{e^{-\frac{r_{2}^{2}}{2}\left(\sqrt{\pi} e^{\frac{r_{2}^{2}}{2}} \operatorname{erf} \frac{r_{2}}{\sqrt{2}}-\sqrt{2} r_{2}\right)}}{\sqrt{2}}-\frac{e^{-\frac{r_{1}^{2}}{2}\left(\sqrt{\pi} e^{\frac{r_{1}^{2}}{2}} \operatorname{erf} \frac{r_{1}}{\sqrt{2}}-\sqrt{2} r_{1}\right)}}{\sqrt{2}}\right]$

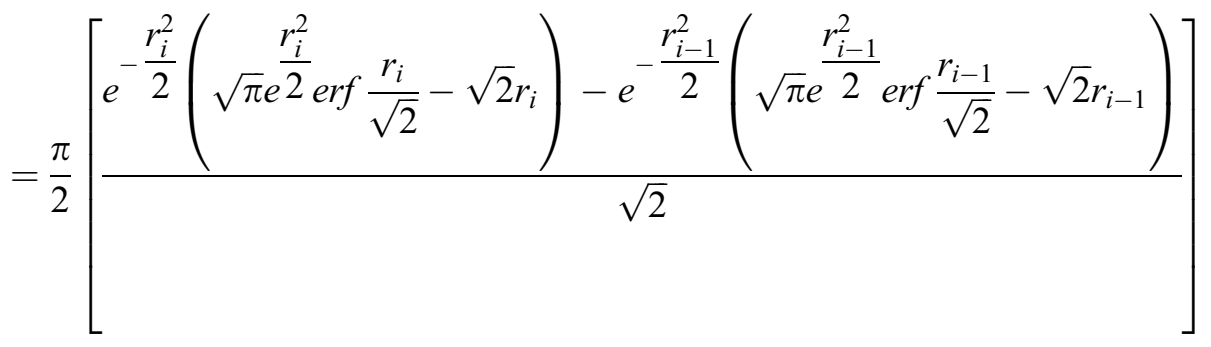


$E\left(t_{\phi}\right)=2 \cdot \frac{1}{2 \pi} \cdot \frac{\pi}{2 \sqrt{2}}\left[\left(\left|r_{i}-r_{i-1}\right|+\frac{\pi}{w_{i}}\right)\left(-r_{i} e^{-\frac{r^{2}}{2}}+\sqrt{\frac{\pi}{2}} \operatorname{erf} \frac{r_{i}}{\sqrt{2}}+r_{i-1} e^{-\frac{r_{i}^{2}}{2}}-\sqrt{\frac{\pi}{2}} \operatorname{erf} \frac{r_{i-1}}{\sqrt{2}}\right)\right]$

$E\left(t_{\phi}\right)=\frac{1}{2 \sqrt{2}}\left[\left|r_{i}-r_{i-1}\right|+\frac{\pi}{w_{i}}\right]\left[-r_{i} e^{-\frac{r_{i}^{2}}{2}}+r_{i-1} e^{-\frac{r_{i-1}^{2}}{2}}+\sqrt{\frac{\pi}{2}}\left(\operatorname{erf} \frac{r_{i}}{\sqrt{2}}-\operatorname{erf} \frac{r_{i-1}}{\sqrt{2}}\right]\right.$.

Special cases:

If $r_{1}-r_{0}=r \rightarrow(3)$, where $r_{0}=0$, then $r_{2}-r_{1}=r \rightarrow(4)$, and $r_{2}=2 r$. Also, $r_{3}-r_{2}=r \Rightarrow r_{3}=3 r$.

Hence,

$E\left(t_{\phi}\right)=\frac{1}{2 \sqrt{2}}\left[r+\frac{\pi}{w_{i}}\right]\left[-r_{i} e^{-\frac{r_{i}^{2}}{2}}+\left(r_{i}-r\right) e^{-\frac{\left(r_{i}-r\right)^{2}}{2}}+\sqrt{\frac{\pi}{2}}\left(\operatorname{erf} \frac{r_{i}}{\sqrt{2}}-\operatorname{erf} \frac{\left(r_{i}-r\right)}{\sqrt{2}}\right)\right]$,

$E\left(t_{\phi}\right)=\frac{1}{2 \sqrt{2}}\left[r+\frac{\pi}{w_{i}}\right]\left[-i r e^{-\frac{(i r)^{2}}{2}}+r(i-1) e^{-\frac{(r(i-r))^{2}}{2}}+\sqrt{\frac{\pi}{2}}\left(\operatorname{erf} \frac{i r}{\sqrt{2}}-\operatorname{erf} \frac{r(i-1)}{\sqrt{2}}\right)\right]$

By considering the values of $\mathrm{i}, \mathrm{r}$, and $w_{i}$ in Tab. 1 using the Mathematica program, and substituting in Eq. (5), we can obtain the different values of $E\left(t_{\phi}\right)$.

Table 1: Expected values of the time taken to detect the lost ship

\begin{tabular}{llll}
\hline $\mathrm{i}$ & $\mathrm{r}$ & $w_{i}$ & $E\left(t_{\phi}\right)$ \\
\hline 0 & 0 & 10 & 0 \\
0 & 1 & 10 & 0.115721 \\
0 & 2 & 10 & 0.978161 \\
0 & 3 & 10 & 1.46451 \\
0 & 4 & 10 & 1.91147 \\
1 & 0 & 10 & 0 \\
1 & 1 & 10 & 0.115721 \\
1 & 2 & 10 & 0.757269 \\
1 & 3 & 10 & 1.42546 \\
1 & 4 & 10 & 1.90942 \\
2 & 0 & 10 & 0 \\
2 & 1 & 10 & 0.314292 \\
\hline & & & (Continued)
\end{tabular}




\begin{tabular}{llll}
\hline \multicolumn{2}{c}{ Table 1 (continued). } & & \\
\hline $\mathrm{i}$ & $\mathrm{r}$ & $w_{i}$ & $E\left(t_{\phi}\right)$ \\
\hline 2 & 2 & 10 & 1.68174 \\
2 & 3 & 10 & 0.0430129 \\
2 & 4 & 10 & 0.000121085 \\
3 & 0 & 10 & 0 \\
3 & 1 & 10 & 0.135183 \\
3 & 2 & 10 & 0.442948 \\
3 & 3 & 10 & 7.03006 \\
3 & 4 & 10 & 0.00409326 \\
4 & 0 & 10 & 0 \\
4 & 1 & 10 & 0.0163944 \\
4 & 2 & 10 & 0.0016467 \\
4 & 3 & 10 & 0.117145 \\
4 & 4 & 10 & 18.3028 \\
0 & 0 & 20 & 0 \\
0 & 1 & 20 & 0.101895 \\
0 & 2 & 20 & 0.911795 \\
0 & 3 & 20 & 1.35793 \\
0 & 4 & 20 & 1.83993 \\
\hline
\end{tabular}

Acknowledgement: We thank the reviewers for careful checking and LetPub (www.letpub.com) for its linguistic assistance during the preparation of this manuscript.

Funding Statement: This research was funded by the Deanship of Scientific Research at Princess Nourah bint Abdulrhman University Fast-track Research Funding Program.

Conflict of Interest: The authors declare that they have no conflicts of interest to report regarding the present study.

\section{References}

[1] A. B. El-Rayes, A. A. Mohamed and H. Fergani, "On the generalized linear search problem," Delta Journal, vol. 6, no. 2, pp. 1-10, 1993.

[2] A. A. Mohamed, "The generalized search for one-dimensional random walker," International Journal of Pure and Applied Mathematics, vol. 19, no. 3, pp. 375-387, 2005.

[3] A. A. Mohamed and M. Abou Gabal, "Generalized optimal search paths for a randomly located target," in Annual Conf., Cairo, Egypt, pp. 17-29, 2000.

[4] A. A. Mohamed and H. M. Abou Gabal, "Linear search with multiple searchers for a randomly moving target," in Int. Conf. for Statistics, Computer Science and Its Application, Egypt, pp. 115-124, 2003.

[5] A. A. Mohamed and H. M. Abou Gabal, "Multiplicative linear search problem," Egyptian Statistical Journal, Cairo University, vol. 48, no. 1, pp. 34-45, 2004. 
[6] Z. T. Balkhi, "The generalized optimal search paths for the continuous univariate random variable," Journal of the Operations Research, vol. 23, no. 1, pp. 67-96, 1989.

[7] A. A. Mohamed, H. M. Abou Gabal and W. A. Afifi, "On the coordinated search problem," International Journal of Applied Mathematics, vol. 25, no. 5, pp. 627-636, 2007.

[8] A. A. Mohamed, H. M. Abou Gabal and W. A. Afifi, "Coordinated search for a randomly located target," International Journal of Contemporary Mathematical Sciences, vol. 8, pp. 5-8, 2013.

[9] A. A. Mohamed, H. M. Abou Gabal and W. A. Afifi, "Generalized coordinated search for a randomly located target," Delta Journal Sciences, vol. 38, pp. 33-42, 2017.

[10] A. Mohamed and W. A. Afifi, "Quasi-coordinate search for a randomly moving target," Journal of Mathematics and Physics, vol. 7, no. 8, pp. 1814-1825, 2019.

[11] W. A. Afifi, A. H. EL-Bagoury and S. N. AL-Aziz, "A novel search algorithm for a multi searchers random walk," Journal of the Applied Mathematics and Information Science, vol. 14, no. 1, pp. 115-122, 2020.

[12] A. Mohamed, H. Fergany and M. El-Hadidy, "On the coordinated search problem on the plane," Journal of the School of Business Administration Istanbul University, vol. 41, no. 1, pp. 80-102, 2012.

[13] M. El-Hadidy and A. H. EL-Bagoury, "Optimal search strategy for a three dimensional randomly located target," International Journal of Operational Research, vol. 29, no. 1, pp. 115-126, 2017.

[14] M. El-Hadidy, A. Teamah and A. H. El-Bagoury, "3-Dimensional coordinated search technique for a randomly located target," International Journal of Computing Science and Mathematics, vol. 9, no. 3, pp. 258-272, 2018.

[15] T. Caraballo, A. A. Teamah and H. EL-Bagoury, "Minimizing the expected time to detect a randomly located lost target using 3-dimensional search technique," Communications in Statistics-Theory and Methods, vol. 49, no. 13, pp. 3313-3328, 2020.

[16] M. A. Kassem, A. H. EL-Bagoury, W. A. Afifi and S. N. AL-Aziz, "On minimum expected search time algorithm for 3-dimensional randomly located target," Journal of Statistics Applications \& Probability, vol. 10, no. 1, pp. 159-166, 2021.

[17] A. A. M. Teamah, W. A. Afifi, J. Gani Dar, A. H. EL-Bagoury and S. N. AL-Aziz, "Optimal discrete search for a randomly moving covid19," Journal of Statistics Applications \& Probability, vol. 9, no. 3, pp. 473-481, 2020.

[18] W. A. Afifi and A. H. EL-Bagoury, "Optimal multiplicative generalized linear search plan for a discrete randomly located target," Information Sciences Letters, vol. 10, no. 1, pp. 153-158, 2021. 